\title{
Strong Quantum Darwinism and Strong Independence are Equivalent to Spectrum Broadcast Structure
}

\author{
Thao P. Le ${ }^{*}$ and Alexandra Olaya-Castro \\ Department of Physics and Astronomy, University College London, \\ Gower Street, London WC1E 6BT, United Kingdom
}

(Received 25 June 2018; published 8 January 2019)

\begin{abstract}
How the objective everyday world emerges from the underlying quantum behavior of its microscopic constituents is an open question at the heart of the foundations of quantum mechanics. Quantum Darwinism and spectrum broadcast structure are two different frameworks providing key insight into this question. Recent works, however, indicate these two frameworks can lead to conflicting predictions on the objectivity of the state of a system interacting with an environment. Here, we provide a resolution to this issue by defining strong quantum Darwinism and proving that it is equivalent to spectrum broadcast structure when combined with strong independence of the subenvironments. We further show that strong quantum Darwinism is sufficient and necessary to signal state objectivity without the requirement of strong independence. Our Letter unveils the deep connection between strong quantum Darwinism and spectrum broadcast structure, thereby making fundamental progress toward understanding and solving the emergence of classicality from the quantum world. Together they provide us a sharper understanding of the transition in terms of state structure, geometry, and quantum and classical information.
\end{abstract}

DOI: $10.1103 /$ PhysRevLett.122.010403

Through the interaction with large environments, quantum systems lose their underlying subjective quantum behavior and appear objective to independent observers. How this transition from the quantum to the classical world happens is not fully understood. Decoherence theory takes a huge leap toward solving this problem [1-3], however, by itself does not explain other important aspects of objectivity such as the redundancy of information. Two different frameworks that can explain this information redundancy are quantum Darwinism [4] and spectrum broadcasting [5]. They are illustrated in Fig. 1. Quantum Darwinism divides the environment into multiple independent fragments and shows that the system-environment interaction can lead to information about the system state being duplicated into such fragments. Quantum Darwinism emerges when multiple different fragments have sufficient information about the system, measured using the quantum mutual information $I(\mathcal{S}: \mathcal{F})$ between system $\mathcal{S}$ and fragment $\mathcal{F}$. Spectrum broadcasting uses a specific classical-quantum state structure called spectrum broadcast structure to signal the emergence of objectivity. These frameworks are complementary in their approach to signal objectivity. The former is entropic in nature, whereas the latter, focused on the state structure, is geometric. Both frameworks have been studied in various spin-spin and spin-boson models [6-19], illuminated spheres [5,20-22], quantum Brownian motion [23-28], single $N$-level environments [29,30], generalized probabilistic theories [31], and even in QED [32], gravitational [33], and experimental quantum-dot [34-38] and photonic [39] settings. Together, quantum Darwinism and spectrum broadcast structure have made important conceptual contributions to the long-standing problem of the quantum-to-classical transition.

Quantum Darwinism and spectrum broadcast structure rely on an agreed definition of objectivity.

Definition: objectivity.-A system state is objective if it is (1) simultaneously accessible to many observers (2) who can all determine the state independently without perturbing it and (3) all arrive at the same result [4,5,9].

For example, our observations of the moon are objectiveby independently observing the light emitted by the moon, different observers can describe the same moon. The definition of what it means to be objective in and of itself is up for debate [this definition can be thought of as intersubjectivity rather than objectivity per se [40] ], but that debate is not purpose of this Letter. For our purpose of understanding when and why quantum Darwinism can be inconsistent with emergence of classical objectivity, this basic definition is sufficient.

Recent works have shown examples in which quantum Darwinism can falsely herald objectivity of the state of a quantum system [5,6,30]. Pleasance and Garraway [6] have considered a qubit coupled to a bosonic environment and found that the mutual information "plateau" that is traditionally used to signal quantum Darwinism - and thus objectivity-was in fact largely composed of quantum discord rather than classical information. We have investigated objectivity in a qubit interacting with an $N$-level environment and have shown that there can be a nonnegligible amount of quantum discord in a situation where 

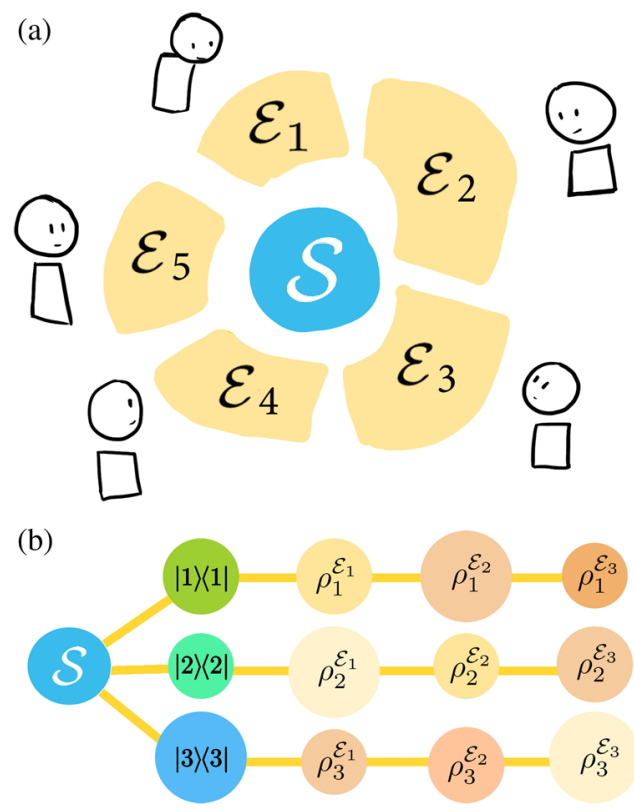

FIG. 1. Quantum Darwinism and spectrum broadcast structure. (a) Quantum Darwinism recognizes that the environment is made up of different fragments, for example, $\mathcal{E}=\mathcal{E}_{1} \otimes \mathcal{E}_{2} \otimes \cdots \otimes \mathcal{E}_{5}$. Different observers access the properties of the system by measuring different environment fragments. (b) A spectrum broadcast structure state can be viewed as the existence of different branches, where there are different, distinguishable states $\left\{\rho_{i}^{\mathcal{E}_{k}}\right\}_{i}$ given different conditional system states.

quantum Darwinism had apparently emerged [30]. Horodecki et al. [5] have argued that certain entangled states could satisfy quantum Darwinism while not being objective. The existence of quantum discord in these cases means that the condition of "measurement without perturbation" will fail, and hence the system state is not objective, despite what quantum Darwinism suggests.

It is precisely in this respect that traditional quantum Darwinism and the spectrum broadcast structure diverge. Spectrum broadcast structure explicitly fulfills the requirement of nonperturbation of measurement in the sense of Bohr nondisturbance [41]. Furthermore, spectrum broadcast structure implies quantum Darwinism, whereas the converse direction does not hold. The mutual information plateau condition of quantum Darwinism is not sufficient to determine whether a state is objective.

Here we propose a resolution to this issue by formulating a stronger version of quantum Darwinism-strong quantum Darwinism. The original mutual information condition is replaced by a stronger condition using the accessible information, Holevo quantity, and the quantum discord. Rather than requiring sufficient mutual information, sufficient classical information, as given by the accessible information and Holevo quantity, is required. The quantum discord must also be vanishing-if the quantum discord is nonzero, then there is information about the system that is not locally accessible by the observer measuring their fragment [42-44]. We prove that strong quantum Darwinism is equivalent to spectrum broadcast structure when combined with strong independence. This leads to the corollary that strong quantum Darwinism is sufficient and necessary for objectivity. In contrast with spectrum broadcast structure, system objectivity does not require strong independence. In its mathematical simplicity, strong Quantum Darwinism makes fundamental progress toward understanding and solving the emergence of classicality from the quantum world. We also suggest an entropic measure for strong quantum Darwinism that complements the geometric distance bound for spectrum broadcast structure [40], unifying the various perspectives used to study the quantum-to-classical transition-state structure, geometric distances, and quantum information theory.

Quantum Darwinism. - For a system-environment state $\left|\Psi_{\mathcal{S E}}\right\rangle$, the reduced density matrix of system is

$$
\rho_{\mathcal{S}}=\operatorname{tr}_{\mathcal{E}}\left[\left|\Psi_{\mathcal{S E}}\right\rangle\left\langle\Psi_{\mathcal{S E}}\right|\right]
$$

Decoherence theory and environment superselection $[45,46]$ describe the preferred pointer basis in which a quantum system decoheres. This is given by the pointer observable $\Pi_{\mathcal{S}}$, that the system will diagonalize under the influence of the environment [45-48]. Because the complete evolution is unitary, quantum Darwinism argues that information about the system $\mathcal{S}$-and in particular, information about the observable $\Pi_{\mathcal{S}}$-must be encoded somewhere in the environment $\mathcal{E}$. This holds for all quantum dynamics regardless of the details of the system-environment interaction $[49,50]$. Observers then obtain information about the system by measuring fragments of the environment. The von Neumann entropy of the system $H(\mathcal{S})=H\left(\rho_{\mathcal{S}}\right)=-\operatorname{tr} \rho_{\mathcal{S}} \log _{2} \rho_{\mathcal{S}}=-\sum_{i} p_{i} \log _{2} p_{i}$, where $p_{i}$ are its eigenvalues, characterizes the information contained within the system. The system is objective from the perspective of the observer if they can obtain precisely this information from their measurement of their environment fragment without perturbing the system. Here, we take the mathematical definition of Bohr nondisturbance [41]: the state remains unchanged after discarding the measurement results of the positive-operator valued measure (POVM) $\left\{M_{i}^{\mathcal{E}_{k}}\right\}$ on any subenvironment $\mathcal{E}_{k}: \sum_{i} M_{i}^{\mathcal{E}_{k}} \rho_{\mathcal{S E}} M_{i}^{\mathcal{E}_{k}^{\dagger}}=\rho_{\mathcal{S E}}$.

Suppose an observer has access to some fragment $\mathcal{F}$ of the environment. The reduced density matrix of system and fragment is

$$
\rho_{\mathcal{S F}}=\operatorname{tr}_{\mathcal{E} \backslash \mathcal{F}}\left[\left|\Psi_{\mathcal{S E}}\right\rangle\left\langle\Psi_{\mathcal{S E}}\right|\right]
$$

where the trace is over all the environment $\mathcal{E}$ except the fragment $\mathcal{F}$. The quantum mutual information, $I(\mathcal{S}: \mathcal{F})=$ $H(\mathcal{S})+H(\mathcal{F})-H(\mathcal{S F})$, measures the total quantum and classical correlations between $\mathcal{S}$ and $\mathcal{F}$. The classically 
accessible information is $I_{\text {acc }}(\mathcal{S}: \mathcal{F})$, and for an objective state, this is equal to the Holevo information on the fragment $\mathcal{F}$ conditioned on the system $\mathcal{S}^{\Pi}$ after measurement of $\Pi_{\mathcal{S}}$ on the system [47]

$\chi\left(\mathcal{S}^{\Pi}: \mathcal{F}\right)=\max _{\hat{\Pi}_{\mathcal{S}}}\left\{H\left(\sum_{a} p_{a} \rho_{\mathcal{F} \mid a}\right)-\sum_{a} p_{a} H\left(\rho_{\mathcal{F} \mid a}\right)\right\}$,

where $a$ are the measurement results of a system POVM $\hat{\Pi}_{\mathcal{S}}, p_{a}$ is the probability of that result, and $\rho_{\mathcal{F} \mid a}$ is the conditional state on the fragment. The Holevo information bounds the maximum information about the classical random variable on the system with probabilities $\left\{p_{a}\right\}$ that can be determined via measurements of the fragment $\mathcal{F}$ [51]. The quantum discord $[42,43]$

$\mathcal{D}\left(\mathcal{S}^{\Pi}: \mathcal{F}\right)=H(\mathcal{S})-H(\mathcal{S F})+\min _{\hat{\Pi}_{\mathcal{S}}} H\left(\sum_{a} p_{a} \rho_{\mathcal{F} \mid a}\right)$,

describes quantum (i.e., nonclassical) correlations beyond entanglement: although entanglement cannot be prepared using local operations and classical communication (LOCC), quantum discord cannot be measured using LOCC [44].

From the complementarity between the classical information in the Holevo quantity with the quantum information given by the quantum discord, the quantum mutual information between system and fragment is in fact $I(\mathcal{S}: \mathcal{F})=\chi\left(\mathcal{S}^{\Pi}: \mathcal{F}\right)+\mathcal{D}\left(\mathcal{S}^{\Pi}: \mathcal{F}\right)[47]$. We have the components required to state strong quantum Darwinism.

Definition: strong quantum Darwinism. - A system state $\mathcal{S}$ is said to be objective when there exists a fragment of the environment $\mathcal{F} \subseteq \mathcal{E}$ such that the following condition holds

$$
I(\mathcal{S}: \mathcal{F})=I_{\text {acc }}(\mathcal{S}: \mathcal{F})=\chi\left(\mathcal{S}^{\Pi}: \mathcal{F}\right)=H(\mathcal{S})
$$

where $I(\mathcal{S}: \mathcal{F})$ is the quantum mutual information, $I_{\text {acc }}(\mathcal{S}: \mathcal{F})$ is the accessible information, $\chi\left(\mathcal{S}^{\Pi}: \mathcal{F}\right)$ is the Holevo quantity in the pointer basis $\Pi$, and $H(\mathcal{S})$ is the von Neumann entropy of the system. For the system state to be objective, Eq. (5) must also hold for multiple disjoint subfragments $\mathcal{F}_{i}$ corresponding to multiple independent observers, where $\mathcal{F}=\mathcal{F}_{1} \cup \mathcal{F}_{2} \cup \ldots \cup \mathcal{F}_{k}, \mathcal{F}_{i} \cap \mathcal{F}_{j}=\varnothing$ for all $i \neq j$.

Strong quantum Darwinism recognizes the difference between shared classical information and shared quantum information: shared quantum information may have nonzero discord and hence information that is not locally accessible. In contrast, traditional quantum Darwinism [4] only requires that $I(\mathcal{S}: \mathcal{F})=H(\mathcal{S})$, and it was assumed that large majority of that information would be classical in nature. However, as the studies of Refs. [5,6,30] show, some of that information can be explicitly quantum in nature. The stronger condition $I(\mathcal{S}: \mathcal{F})=\chi\left(\mathcal{S}^{\Pi}: \mathcal{F}\right)$ is also assumed by Zwolak and Zurek [47], which they call

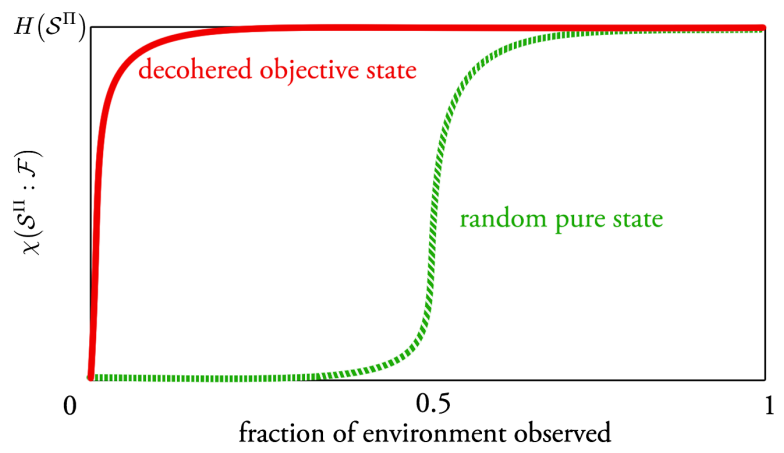

FIG. 2. Accessible classical information given by the Holevo information $\chi\left(\mathcal{S}^{\Pi}: \mathcal{F}\right)$ about the system stored in different fractions of the environment. (Green dashed) For pure states picked out at random (through a Haar measure), the amount of accessible classical information and quantum discord are typically roughly the same. (Red solid) For a state that satisfies strong quantum Darwinism, such as the reduced GHZ state, $\chi\left(\mathcal{S}^{\Pi}: \mathcal{F}\right)$ will be approximately equal to the system entropy $H(\mathcal{S})$ for small fractions of the environment, suggesting the existence of multiple copies of the information and hence redundancy [cf. analogous Fig. 2 in Zurek [4] ].

"surplus decoherence", but was not rigorously imposed as a core part of quantum Darwinism as we have done here.

To define the redundancy and spread of the classical information, suppose there are fragments $\mathcal{F}_{\delta}$ with size $\left|\mathcal{F}_{\delta}\right|=f_{\delta}|\mathcal{E}|$ that contain classical information

$$
I(\mathcal{S}: \mathcal{F}) \approx \chi\left(\mathcal{S}^{\Pi}: \mathcal{F}\right) \geq(1-\delta) H\left(\mathcal{S}^{\Pi}\right),
$$

that is approximately the information about the system. The redundancy $R_{\delta}$ is the number of unique copies of that information, i.e., the number of disjoint fragments $\mathcal{F}_{\delta, i}$ (where $i$ indices different fragments) that contain that approximate information, $R_{\delta}=\left|\mathfrak{F}_{\delta}\right|$, where

$$
\mathfrak{F}_{\delta}=\left\{\begin{array}{l|l}
\mathcal{F}_{\delta, i} & \begin{array}{c}
\chi\left(\mathcal{S}^{\Pi}: \mathcal{F}_{\delta, i}\right) \geq(1-\delta) H\left(\mathcal{S}^{\Pi}\right), \\
\mathcal{F}_{\delta, i} \cap \mathcal{F}_{\delta, j}=\varnothing \quad \forall i \neq j
\end{array}
\end{array}\right\} .
$$

This is bounded by the minimum fraction size $f_{\delta, \min }$ : $R_{\delta} \leq 1 / f_{\delta \text {,min }}$. If $\chi\left(\mathcal{S}^{\Pi}: \mathcal{F}\right) \geq(1-\delta) H\left(\mathcal{S}^{\Pi}\right)$, then the discord is bounded by $\mathcal{D}\left(\mathcal{S}^{\Pi}: \mathcal{F}\right) \leq \delta H\left(\mathcal{S}^{\Pi}\right)$.

A rapid rise of the classical information $\chi\left(\mathcal{S}^{\Pi}: \mathcal{F}\right)$, as shown schematically in Fig. 2, implies that only a small fraction $f_{\delta}$ of the environment is required to have access to all the information in the system and hence suggesting that there is a large redundancy $R_{\delta} \approx 1 / f_{\delta}$; this occurs for postdecohered system-environment states (that have spectrum broadcast structure). In contrast, Haar-random pure system-environment states [52] will tend to have a mixture of classical and quantum correlations between any system and fragment, and a fairly large fraction would be required to access any substantial amount of information about the system. 
Now, we present the definitions for spectrum broadcast structure and strong independence [5]:

Definition: spectrum broadcast structure.-The joint state $\rho_{\mathcal{S F}}$ of the system $\mathcal{S}$ and a collection of subenvironments $\mathcal{F}=\mathcal{E}_{1} \otimes \ldots \otimes \mathcal{E}_{F}$ have spectrum broadcast structure if it can be written as

$$
\rho_{\mathcal{S F}}=\sum_{i} p_{i}|i\rangle_{\mathcal{S}}\langle i| \otimes \rho_{i}^{\mathcal{E}_{1}} \otimes \cdots \otimes \rho_{i}^{\mathcal{E}_{F}},
$$

where $\{|i\rangle\}$ is the pointer basis, $p_{i}$ are probabilities, and all states $\rho_{i}^{\mathcal{E}_{k}}$ are perfectly distinguishable: $\rho_{i}^{\mathcal{E}_{k}} \rho_{j}^{\mathcal{E}_{k}}=0$ $\forall i \neq j$, for each observed environment $\mathcal{E}_{k} \in$ $\left\{\mathcal{E}_{1}, \ldots, \mathcal{E}_{F}\right\}[5]$.

Definition: strong independence.-Subenvironments $\mathcal{E}_{j} \in\left\{\mathcal{E}_{1}, \mathcal{E}_{2}, \ldots, \mathcal{E}_{F}\right\}$ have strong independence relative to the system $\mathcal{S}$ if their conditional mutual information is vanishing

$$
I\left(\mathcal{E}_{j}: \mathcal{E}_{k} \mid \mathcal{S}\right)=0 \quad \forall j \neq k .
$$

Strong independence means that there are no correlations between the environments conditioned on the information about the system.

With strong quantum Darwinism, strong independence, and spectrum broadcast structure now defined, we can prove the titular theorem of this Letter.

Theorem.-A state $\rho_{\mathcal{S F}}$ has spectrum broadcast structure if and only if it satisfies strong quantum Darwinism and has strong independence.

The complete proof is given in Appendix A of the Supplemental Material [53]. In the forward direction $(\Rightarrow)$, an explicit form of the state $\rho_{\mathcal{S F}}$ with spectrum broadcast structure is used to calculate the various mutual and accessible information as well as the entropies required to fulfill strong quantum Darwinism. Spectrum broadcast structure explicitly satisfies strong independence [5]. In the reverse direction $(\Leftarrow)$, the conditions of strong quantum Darwinism [including surplus decoherence of Ref. [47] and classical-only correlations of Ref. [55] ] enforce particular structures on the state $\rho_{\mathcal{S F}}$, and we use these properties and general properties of states, to recover bipartite spectrum broadcast structure, $\rho_{\mathcal{S F}}=\sum_{i} p_{i}|i\rangle_{\mathcal{S}}\langle i| \otimes \rho_{i}^{\mathcal{F}}$, where $\rho_{i}^{\mathcal{F}}$ are mutually distinguishable. Applying strong independence recovers the form in Eq. (8).

Corollary 1.-Strong quantum Darwinism is equivalent to bipartite spectrum broadcast structure.

This proof comes from the proof of main theorem: the result has been encapsulated into a corollary in order to prove the following statement about objectivity:

Corollary 2.-Strong quantum Darwinism is sufficient and necessary for objectivity

$$
\left(\begin{array}{c}
\text { strong } \\
\text { quantum Darwinism }
\end{array}\right) \Leftrightarrow \text { (objectivity). }
$$

Proof.-Horodecki et al. [5] prove precisely Eq. (10) for bipartite spectrum broadcast structure (in place of strong quantum Darwinism). By Corollary 1, Eq. (10) holds for strong quantum Darwinism.

Remark.-In fact, Horodecki et al. [5] proved that objectivity + strong independence $\Rightarrow$ spectrum broadcast structure. Hence, spectrum broadcast structure is an overly stringent requirement for objectivity, in contrast to strong quantum Darwinism which is both sufficient and necessary without additives. Strong independence itself is not required for the system objectivity: in Appendix B of the Supplemental Material [53], we provide examples of objective states that exhibit bipartite broadcast structure, but not strong independence.

Measuring strong quantum Darwinism.- Through the lens of strong quantum Darwinism, a large Holevo quantity $\chi\left(\mathcal{S}^{\Pi}: \mathcal{F}\right)$ is required for objectivity, while the discord $\mathcal{D}\left(\mathcal{S}^{\Pi}: \mathcal{F}\right)$ is a hindrance. As such, we suggest the following as a measure that captures the degree of objectivity of a state in the presence of discord

$$
M^{\mathrm{SQD}}\left(\rho_{\mathcal{S F}}\right) \equiv \frac{H(\mathcal{S})-\chi\left(\mathcal{S}^{\Pi}: \mathcal{F}\right)+\mathcal{D}\left(\mathcal{S}^{\Pi}: \mathcal{F}\right)}{2 H(\mathcal{S})}
$$

which takes values between $[0,1]$. Objectivity occurs when the minimum value is obtained, $M^{\mathrm{SQD}}\left(\rho_{\mathcal{S F}}\right)=0$ signaling perfect strong quantum Darwinism. This measure is not unique, implying a certain partial ordering of states. The components of Eq. (5) can be combined to form other valid measures with different orderings.

Mironowicz et al. [40] have defined a geometric distance bound on how close a state $\rho_{\mathcal{S F}}$ is to being of spectrum broadcast structure

$$
T^{\mathrm{SBS}}\left(\rho_{\mathcal{S F}}\right)=\frac{1}{2} \min _{\Pi_{\mathcal{S}}}\left\|\rho_{\mathcal{S F}}-\rho_{\mathcal{S}^{\Pi} \mathcal{F}}^{\mathrm{SBS}}\right\|_{1} \leq \eta\left(\rho_{\mathcal{S F}}\right),
$$

where

$$
\begin{aligned}
\eta\left(\rho_{\mathcal{S F}}\right) \equiv & \left\|\rho_{\mathcal{S F}}-\rho_{\mathcal{S}^{\Pi} \mathcal{F}}\right\|_{1} \\
& +\sum_{i \neq j} \sqrt{p_{i} p_{j}} B\left(\rho_{\mathcal{F} \mid i}, \rho_{\mathcal{F} \mid j}\right) .
\end{aligned}
$$

The fidelity is $B\left(\rho_{1}, \rho_{2}\right)=\left\|\sqrt{\rho_{1}} \sqrt{\rho_{2}}\right\|_{1}$, and $\rho_{\mathcal{S}^{\Pi} \mathcal{F}}=$ $\sum_{i} p_{i}|i\rangle\langle i| \otimes \rho_{\mathcal{F} \mid i}$ is the postmeasurement (separable) state. Notice that our proposed $M^{\mathrm{SQD}}\left(\rho_{\mathcal{S F}}\right)$ and Mironowicz et al. [40] $\eta\left(\rho_{\mathcal{S F}}\right)$ are related to each other. The term $\left\|\rho_{\mathcal{S F}}-\rho_{\mathcal{S}^{\Pi} \mathcal{F}}\right\|_{1}=\mathcal{D}_{\text {geo }}\left(\mathcal{S}^{\Pi}: \mathcal{F}\right)$ is the geometric quantum discord [56]; hence, it is related to the entropic quantum discord. For two-qubit states, we have the explicit bound with the entropic quantum discord: $\left\|\rho_{\mathcal{S F}}-\rho_{\mathcal{S}^{\Pi} \mathcal{F}}\right\|_{1} \geq$ $\sqrt{2} \mathcal{D}\left(\mathcal{S}^{\Pi}: \mathcal{F}\right) \geq \mathcal{D}\left(\mathcal{S}^{\Pi}: \mathcal{F}\right)$ [57-60]. Similarly, for a qubit system, the Holevo quantity is bounded as $\chi\left(\mathcal{S}^{\Pi}: \mathcal{F}\right) \geq$ $I_{\text {acc }}(\mathcal{S}: \mathcal{F}) \geq H\left(\left\{p_{1}, p_{2}\right\}\right)-2 \sqrt{p_{1} p_{2}} B\left(\rho_{\mathcal{F} \mid 1}, \rho_{\mathcal{F}: 2}\right)$ 
[Eq. (5) in Ref. [61] ]. Hence, in the case where the systemfragment $\rho_{\mathcal{S F}}$ is a two-qubit state

$$
\begin{aligned}
\eta\left(\rho_{\mathcal{S F}}\right) & \geq \mathcal{D}\left(\mathcal{S}^{\Pi}: \mathcal{F}\right)-\chi\left(\mathcal{S}^{\Pi}: \mathcal{F}\right)+H\left(\left\{p_{1}, p_{2}\right\}\right) \\
& =2 H(\mathcal{S}) M^{\mathrm{SQD}}\left(\rho_{\mathcal{S F}}\right) .
\end{aligned}
$$

The calculation of the entropic quantities of strong quantum Darwinism requires optimization over measurements on the system. Without the use of the computable bound $\eta\left(\rho_{\mathcal{S F}}\right)$, calculating the distance to the set of spectrum broadcasting states would require optimization over both the system and all the subenvironments bases.

Discussion. - We have shown a fundamental shift in understanding the emergence of classicality through classical information redundancy as opposed to more general quantum information redundancy. We introduced strong quantum Darwinism by identifying that shared classical information is required for objectivity and by noting that the existence of quantum correlations hinders objectivity. Formally, we examined the nature of the shared system-environment information using the tools of quantum information theory. By proving that the combined strong quantum Darwinism and strong independence is equivalent to spectrum broadcast structure, we have provided a sharper understanding of the quantum-to-classical transition: strong quantum Darwinism alone is necessary and sufficient for objectivity of a system state, capturing succinctly the minimal requirements of objectivity. In contrast, spectrum broadcast structure describes both objectivity of the system state and partial objectivity of the environment states. Finally, we have suggested a possible measure for the degree of objectivity using classical and quantum information, complementing the state structure and geometric perspectives of spectrum broadcasting.

The discrepancy between the classical accessible information and quantum mutual information observed in earlier papers $[6,30]$ is now resolved: the discrepancy implies that strong quantum Darwinism does not emerge, and there is no objectivity nor spectrum broadcast structure. Strong quantum Darwinism also addresses the concerns by Horodecki et al. [5], whereby traditional quantum Darwinism emerges even when the systemenvironment state was clearly entangled. The example in Ref. [5] is the following:

$$
\rho_{\mathcal{S E}}=p P_{(a|00\rangle+b|11\rangle)}+(1-p) P_{(a|10\rangle+b|01\rangle)},
$$

where $P_{|\psi\rangle}=|\psi\rangle\langle\psi|, p \neq 1 / 2, a=\sqrt{p}$, and $b=\sqrt{1-p}$. In Appendix $C$ of the Supplemental Material [53], we determine that $I(\mathcal{S}: \mathcal{E})=H(\mathcal{S})$; i.e., quantum Darwinism is satisfied while $\mathcal{X}\left(\mathcal{S}^{\Pi}: \mathcal{E}\right) \neq H(\mathcal{S})$ for $p \neq 0$, 1. Strong quantum Darwinism is not satisfied in general, and this is consistent with the correct conclusion that the system is not objective.

Strong quantum Darwinism opens up yet further questions to be addressed. We have shown that strong quantum Darwinism deviates from spectrum broadcast structure when there are intra-subenvironmental correlations. Then, when does strong quantum Darwinism deviate from the usual quantum Darwinism? Many past studies of models in the literature find quantum Darwinism also assume no system self-Hamiltonian, or that the system Hamiltonian $H_{S}$ commutes with the coupling Hamiltonian $H_{I}$. In contrast, the two examples where strong quantum Darwinism is needed $[6,30]$, both have that $\left[H_{S}, H_{I}\right] \neq 0$ yet with very different kinds of system-bath Hamiltonians. The work of Ref. [62] shows that the commuting properties of $H_{S}$ and $H_{I}$ can shape the nature of the correlations, work, entropy, etc. in bipartite systems. These examples also displayed strong system-environment correlations and non-Markovian dynamics. Therefore, we conjecture that strong zquantum Darwinism deviates from traditional quantum Darwinism when there is a non-negligible self-Hamiltonian, a coupling Hamiltonian that does not commute with it, and strong system-environment correlations.

The quantum-to-classical transition remains an unsolved problem. Strong quantum Darwinism captures, formally and conceptually, the essence of what will be required: the emergence of perfect classical correlations and the disintegration of quantum correlations between objective objects and independent observers.

Upon completion of our Letter, two experimental works have been reported investigating quantum Darwinism in photonic [63] and spin [64] environments, which base their analysis in the Holevo information. Our Letter and the newly introduced concept of strong quantum Darwinism give solid foundation to these experimental works.

We thank R. Horodecki, J. Korbicz, and P. Horodecki for discussions. We thank the anonymous referees for their constructive feedback. This work was supported by the Engineering and Physical Sciences Research Council (Grant No. EP/L015242/1).

*thao.le.16@ucl.ac.uk †a.olaya@ucl.ac.uk

[1] E. Joos and H. D. Zeh, Z. Phys. B 59, 223 (1985).

[2] M. Schlosshauer, Rev. Mod. Phys. 76, 1267 (2005).

[3] M. A. Schlosshauer, Decoherence and the Quantum-ToClassical Transition (Springer, Berlin, Heidelberg, 2007).

[4] W. H. Zurek, Nat. Phys. 5, 181 (2009).

[5] R. Horodecki, J. K. Korbicz, and P. Horodecki, Phys. Rev. A 91, 032122 (2015).

[6] G. Pleasance and B. M. Garraway, Phys. Rev. A 96, 062105 (2017).

[7] N. Balanesković, Eur. Phys. J. D 69, 232 (2015).

[8] N. Balanesković and M. Mendler, Eur. Phys. J. D 70, 177 (2016). 
[9] H. Ollivier, D. Poulin, and W. H. Zurek, Phys. Rev. Lett. 93, 220401 (2004).

[10] R. Blume-Kohout and W. H. Zurek, Found. Phys. 35, 1857 (2005).

[11] C. J. Riedel, W. H. Zurek, and M. Zwolak, New J. Phys. 14, 083010 (2012).

[12] M. Zwolak, C. J. Riedel, and W. H. Zurek, Sci. Rep. 6, 25277 (2016).

[13] M. Zwolak, H. T. Quan, and W. H. Zurek, Phys. Rev. Lett. 103, 110402 (2009).

[14] M. Zwolak, H. T. Quan, and W. H. Zurek, Phys. Rev. A 81, 062110 (2010).

[15] G. L. Giorgi, F. Galve, and R. Zambrini, Phys. Rev. A 92 , 022105 (2015).

[16] M. Zwolak, C. J. Riedel, and W. H. Zurek, Phys. Rev. Lett. 112, 140406 (2014).

[17] M. Zwolak and W. H. Zurek, Phys. Rev. A 95, 030101 (2017).

[18] A. Lampo, J. Tuziemski, M. Lewenstein, and J. K. Korbicz, Phys. Rev. A 96, 012120 (2017).

[19] P. Mironowicz, P. Należyty, P. Horodecki, and J. K. Korbicz, Phys. Rev. A 98, 022124 (2018).

[20] C. J. Riedel and W. H. Zurek, Phys. Rev. Lett. 105, 020404 (2010).

[21] C. J. Riedel and W. H. Zurek, New J. Phys. 13, 073038 (2011).

[22] J. K. Korbicz, P. Horodecki, and R. Horodecki, Phys. Rev. Lett. 112, 120402 (2014).

[23] J. Tuziemski and J. K. Korbicz, Europhys. Lett. 112, 40008 (2015).

[24] J. Tuziemski and J. Korbicz, Photonics 2, 228 (2015).

[25] J. Tuziemski and J. K. Korbicz, J. Phys. A 49, 445301 (2016).

[26] F. Galve, R. Zambrini, and S. Maniscalco, Sci. Rep. 6, 19607 (2016).

[27] R. Blume-Kohout and W. H. Zurek, Phys. Rev. Lett. 101, 240405 (2008).

[28] J. P. Paz and A. J. Roncaglia, Phys. Rev. A 80, 042111 (2009).

[29] A. Pérez, Phys. Rev. A 81, 052326 (2010).

[30] T. P. Le and A. Olaya-Castro, Phys. Rev. A 98, 032103 (2018).

[31] C. M. Scandolo, R. Salazar, J. K. Korbicz, and P. Horodecki, arXiv:1805.12126.

[32] J. Tuziemski, P. Witas, and J. K. Korbicz, Phys. Rev. A 97, 012110 (2018).

[33] J. K. Korbicz and J. Tuziemski, Gen. Relativ. Gravit. 49, 152 (2017).

[34] R. Brunner, R. Akis, D. K. Ferry, F. Kuchar, and R. Meisels, Phys. Rev. Lett. 101, 024102 (2008).

[35] R. Brunner, R. Akis, R. Meisels, F. Kuchar, and D. Ferry, Physica (Amsterdam) 42E, 1208 (2010).

[36] R. Brunner, D. K. Ferry, R. Akis, R. Meisels, F. Kuchar, A. M. Burke, and J. P. Bird, J. Phys. Condens. Matter 24, 343202 (2012).
[37] A. M. Burke, R. Akis, T. E. Day, G. Speyer, D. K. Ferry, and B. R. Bennett, Phys. Rev. Lett. 104, 176801 (2010).

[38] D. K. Ferry, R. Akis, and R. Brunner, Phys. Scr. T165, 014010 (2015).

[39] M. A. Ciampini, G. Pinna, P. Mataloni, and M. Paternostro, Phys. Rev. A 98, 020101 (2018).

[40] P. Mironowicz, J. K. Korbicz, and P. Horodecki, Phys. Rev. Lett. 118, 150501 (2017).

[41] N. Bohr, Phys. Rev. 48, 696 (1935).

[42] H. Ollivier and W. H. Zurek, Phys. Rev. Lett. 88, 017901 (2001).

[43] L. Henderson and V. Vedral, J. Phys. A 34, 6899 (2001).

[44] K. Modi, Open Syst. Inf. Dyn. 21, 1440006 (2014).

[45] W. H. Zurek, Prog. Theor. Phys. 89, 281 (1993).

[46] W. H. Zurek, Rev. Mod. Phys. 75, 715 (2003).

[47] M. Zwolak and W. H. Zurek, Sci. Rep. 3, 1729 (2013).

[48] W. Zurek, Ann. Phys. (N.Y.) 9, 855 (2000).

[49] F. G. S. L. Brandão, M. Piani, and P. Horodecki, Nat. Commun. 6, 7908 (2015).

[50] P. A. Knott, T. Tufarelli, M. Piani, and G. Adesso, Phys. Rev. Lett. 121, 160401 (2018).

[51] A. S. Holevo, Prob. Peredachi Inf. 9, 3 (1973).

[52] For example, a Haar-random pure state $\left|\psi_{\mathcal{S E}}\right\rangle=U\left|\psi_{0}\right\rangle$ can be realized using some reference state $\left|\psi_{0}\right\rangle$ and a random unitary matrix $U$ drawn with a uniform probability from the set of all unitary matrices on the system and environment.

[53] See Supplemental Material at http://link.aps.org/ supplemental/10.1103/PhysRevLett.122.010403 for a proof of the main theorem, examples of an objective system state without strong independence and of an entangled state that statisfies quantum Darwinism but not strong quantum Darwinism, which includes Ref. [54].

[54] M. B. Ruskai, J. Math. Phys. (N.Y.) 43, 4358 (2002).

[55] M. Piani, P. Horodecki, and R. Horodecki, Phys. Rev. Lett. 100, 090502 (2008).

[56] B. Dakić, V. Vedral, and Č. Brukner, Phys. Rev. Lett. 105, 190502 (2010).

[57] D. Girolami and G. Adesso, Phys. Rev. A 83, 052108 (2011).

[58] D. Girolami and G. Adesso, Phys. Rev. A 84, 052110 (2011).

[59] F. M. Paula, T. R. de Oliveira, and M. S. Sarandy, Phys. Rev. A 87, 064101 (2013).

[60] W. Roga, D. Spehner, and F. Illuminati, J. Phys. A 49, 235301 (2016).

[61] R. Jain and A. Nayak, arXiv:quant-ph/0603278v4.

[62] H. Hossein-Nejad, E. J. O’Reilly, and A. Olaya-Castro, New J. Phys. 17, 075014 (2015).

[63] M.-C. Chen, H.-S. Zhong, Y. Li, D. Wu, X.-L. Wang, L. Li, N.-L. Liu, C.-Y. Lu, and J.-W. Pan, arXiv:1808.07388v1.

[64] T. Unden, D. Louzon, M. Zwolak, W. H. Zurek, and F. Jelezko, arXiv:1809.10456v2. 